\title{
Personalized Immuno-Oncology
}

\author{
Kewal K. Jain \\ Jain PharmaBiotech, Basel, Switzerland
}

\section{Highlights}

- Modulation of the immune system is important in the management of cancer.

- Anticancer strategies in immuno-oncology include pharmaceuticals such as immune checkpoint inhibitors and biological therapies such as cell therapy and vaccines.

- As anticancer immune responses can be modulated in several ways that vary from patient to patient, immunotherapy needs to be personalized for individual patients.

- This review highlights how immuno-oncology is personalized.

\section{Keywords}

Cell-based immune therapies · Immune checkpoint inhibitors - Immunogene therapy - Immuno-oncology . Personalized cancer vaccines . Personalized oncology . Precision oncology

\begin{abstract}
Cancer immunotherapy, which aims to control the immune system to eradicate cancer cells and prevent their spread, needs to be personalized because anticancer immune responses can be inhibited in several ways that vary from patient to patient. Cancer immunotherapy includes pharmaceuticals such as immune checkpoint inhibitors and monoclonal antibodies (MAbs) as well as cell therapy, immunogene therapy, and vaccines. Combination of programmed cell death protein 1 (PD-1)/programmed cell death protein ligand 1 (PD-L1) drugs with other immunotherapy drugs, for example, antibody-drug conjugates, as well as combination of PD-1/PD-L1 drugs with other therapies, for example, chemotherapy and radiation therapy, are being explored. Biomarkers are important for predicting the response to immu-
\end{abstract}

karger@karger.com www.karger.com/mpp

Karger $\stackrel{\text { ' }}{=}$

GOPEN ACCESS
(C) 2020 The Author(s)

Published by S. Karger AG, Basel

This is an Open Access article licensed under the Creative Commons Attribution-NonCommercial-4.0 International License (CC BY-NC) (http://www.karger.com/Services/OpenAccessLicense), applicable to the online version of the article only. Usage and distribution for commercial purposes requires written permission. notherapy. Molecular diagnostics and sequencing are important technologies for guiding treatment in immunooncology. Genomic profiling of tumor mutational burden may enhance the predictive utility of PD-L1 expression and facilitate personalized combination immunotherapy. Optimization of personalized immuno-oncology requires integration of several technologies and selection of those best suited for an individual patient. Advances in immuno-oncology are also attributed to technologies for targeted delivery of anticancer therapeutics such as antigen-capturing nanoparticles for precision targeting and selective delivery. A breakthrough in cell therapy of cancer is a chimeric antigen receptors- $T$ cell, which combines the antigen-binding site of a MAb with the signal activating machinery of a T cell, freeing antigen recognition from major histocompatibility complex restriction. Gene-editing tools such as clustered regularly interspaced short palindromic repeats have a promising application for removing alloreactivity and decreasing immunogenicity of third-party $\mathrm{T}$ cells. In conclusion, personalized immuno-oncology is one of the most promising approaches to management of cancer.

(C) 2020 The Author(s)

Published by S. Karger AG, Basel

Kewal K. Jain

Jain PharmaBiotech

Bläsiring 7

$\mathrm{CH}-4057$ Basel (Switzerland)

jain@ pharmabiotech.ch 


\section{Introduction to Personalized Medicine}

Personalized medicine, also referred to as individualized medicine, simply means the prescription of specific treatments and therapeutics best suited for an individual, taking into consideration both genetic and environmental factors that influence response to therapy [1]. The term "precision medicine" is used because diagnostic, prognostic, and therapeutic strategies are precisely tailored to each patient's requirements. However, it does not cover the full scope of personalized medicine. Genomic/ proteomic technologies have facilitated the development of personalized medicines, but other technologies such as metabolomics are also contributing to this effort. Personalized medicine is the best way to integrate new biotechnologies into medicine for improving the understanding of pathomechanism of diseases and management of patients. The most important area for application of personalized medicine is cancer, not only because of the variations among patients, but also among tumors with the same histological diagnosis.

\section{Immuno-Oncology}

Cancer immunotherapy aims to control the immune system to eradicate cancer cells and prevent their spread. Caution is exercised in manipulating the immune system, which has potentially dual functions (double-edged sword), that is, promoting tumor development on the one hand and restraining tumor development on the other hand. Currently, most of the applications for new cancer drugs submitted to the FDA in the USA are for immunotherapies or combinations involving immunotherapies. Some of the basic aspects described in the next 2 sections indicate that immuno-oncology is already being personalized.

\section{Classification of Therapeutic Approaches in Immuno-Oncology}

A classification of various therapeutic approaches to cancer immunotherapy is shown in Table 1. Old immunotherapies were passive therapies defined as immunology-based treatments that do not engage the patient's immune system directly. They include antibodies and cell therapies, also known as adoptive cell transfer (ACT). In active immuno-oncology, the patient's own immune system is stimulated with the use of an antigen-presenting cell (APC). The immune system recognizes the APC as an invader and mounts an immune response. Active therapies include cytokine treatments, therapeutic vaccines, immune checkpoint inhibitors (ICIs), and small molecules.

\section{Role of Biomarkers in Personalized Immuno- Oncology}

Biomarkers serve the following purposes in personalizing immuno-oncology:

- Identification of molecular targets as predictive biomarkers is important for matched targeted therapy.

- Integration of biomarkers in clinical trial designs.

- Constant evaluation of the roles of biomarkers and matched targeted therapies is needed during the development and even after approval.

- Identification and validation of biomarkers to predict response to combination therapies, including those that characterize the tumor microenvironment and targeted signaling pathways.

Technologies used for biomarkers used in ICI therapy include immunohistochemistry, genomics, expression signatures, multiplex fluorescence, and circulating biomarkers. Programmed cell death protein ligand 1 (PDL1) is a biomarker for advanced triple-negative breast cancer (TNBC), which is defined as a tumor that lacks expression of estrogen receptor, progesterone receptor, and human EGFR2. In patients with treatment-naive metastatic TNBC, the combination of atezolizumab and nab-paclitaxel is effective with survival benefit if the expression of programmed cell death protein 1 (PD-1) is $\geq 1 \%$ [2]. Resistance to PD-1 blockade is a challenge for some cancer patients, and no validated biomarkers are available for guiding therapy. Emerging predictive biomarkers for immune checkpoint blockade are PD-L1 expression, increased amounts of tumor-infiltrating lymphocytes (TILs), increased mutational load, and mismatch repair deficiency [3]. Other well-studied biomarkers include inflammatory infiltrate, absolute lymphocyte count, and lactate dehydrogenase levels. Currently available tests do not reliably classify the expression of immune cell PD-L1 protein, but efforts are in progress to improve the testing.

Measuring the pharmacological effects of immune checkpoint blockers requires functional assessment of both immune cells and cancer cells. The development path of a companion diagnostic is guided by biological rationale. To establish a biomarker's biological rationale 
Table 1. Classification of various therapeutic approaches to cancer immunotherapy

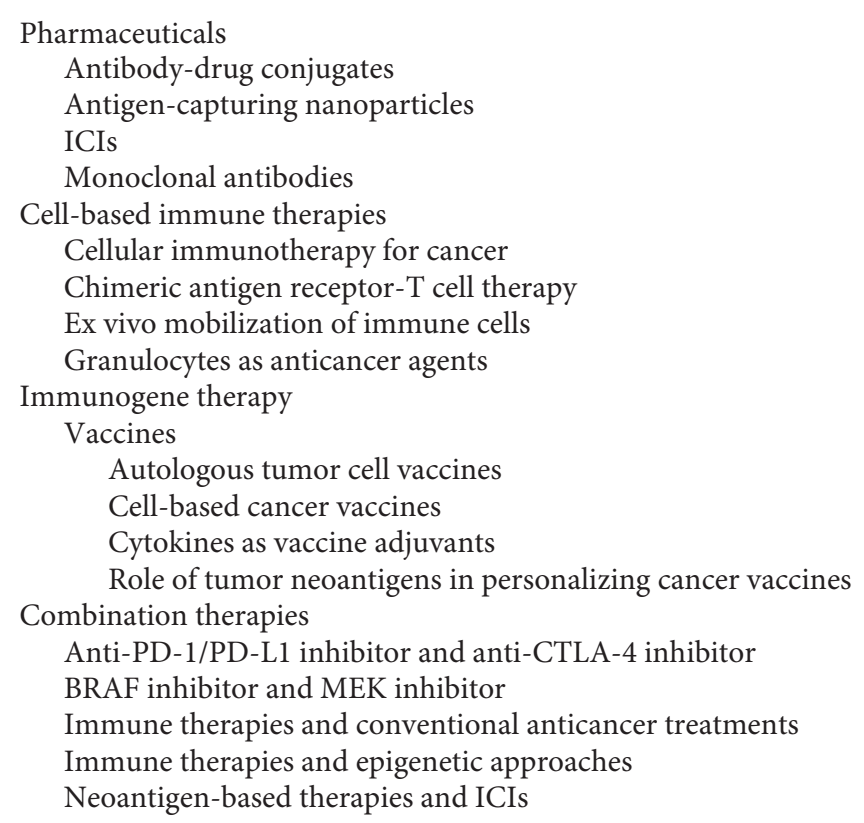

PD-1, programmed cell death protein 1; PD-L1, programmed cell death protein ligand 1; ICI, immune checkpoint inhibitor; CTLA-4, cytotoxic T-lymphocyte-associated protein 4 .

and potential clinical utility in cancer, it is important to determine whether there exists a molecular signature that predicts pathology or the response to therapy, for example, enhancement of $\mathrm{T}$ cell activation to elicit an antitumor immune response is a goal of several new investigational drugs. Data-rich informative biomarker panels that transform traditional immunohistochemistry from visual morphology to data-based analysis are needed. Detection and quantification of biomarkers, as well as gaining an understanding of their spatial relationships, is the first step in analytical verification of a multiplex immunohistochemistry assay. A multiplex immunofluorescence assay has been used to assess anti-PD-1 therapy, which is predicted to enhance $\mathrm{T}$ cell cytotoxic activity, as demonstrated by an increased number of TILs and elevated Zap70-pY493 expression [4]. This assay is being used for pharmacodynamic evaluations in ongoing immunotherapy clinical trials.

Using the methods of International Immuno-Oncology Biomarker Working Group, a study has correlated the levels of TILs with clinicopathological features in different malignancies [5]. TIL levels in this study were higher in invasive margin compartments, intratumoral and stro- mal compartments, and predicted survival. Cutaneous melanoma had the highest density of lymphocyte and macrophage populations, whereas CD20 density was associated with survival in the whole series.

Biomarkers such as tumor mutational burden (TMB) and microsatellite instability are useful for stratification of patients for targeted immuno-oncology therapies, which require extensive genomic profiling for sensitive detection of clinically relevant variants. Detection of single and multiple nucleotide variants - CNVs, microsatellite instability, and TMB - has been evaluated using a commercially available next generation sequencing (NGS) panel containing 523 cancer-related genes and can be applied for detection of multiple DNA-based biomarkers relevant for treatment selection [6].

\section{Role of Organoids in Predicting Response to Immuno-Therapeutic Drugs}

Organoids are 3D cultures of cancer cells, also referred to as organotypic tumor spheroid cultures, which can be grown from tumor biopsies and recapitulate tumor heterogeneity as well as maintain genomic integrity better than cell lines. As ex vivo systems that incorporate features of the tumor microenvironment, they can be used to model the dynamic response to ICIs. Tumor organoids may enable evaluation of response to therapy to select patients who are likely to respond to immunotherapy and avoid treatment-related complications in non-responders [7]. They may facilitate the selection of an effective combination of therapies as part of personalized immuno-oncology approach. However, there are some limitations to the use of cancer organoids due to their in vitro nature, as they lack vascularity, which is a characteristic of most tumors [8]. Despite this limitation, another study has shown that organoid-drug interactions accurately predict and mirror patient drug response in vivo [9].

\section{Role of Tumor-On-Chips in Immuno-Oncology}

Advantages of tumor-on-chips on other in vitro models, such as classical cultures and spheroids, are a controlled 3D architecture and dynamic microflow conditions, with exchange of oxygen and nutrients enabling operation for up to several days. Reduced thickness and greater homogeneity as compared to spheroids facilitate real-time imaging as well as access of a drug to tumor cells [10]. Constructing a complex immune system on a chip 
is a daunting task that has not been achieved, but immune cells can be added to the tumor either as an adjacent extracellular compartment or perfused through a "surrogate blood vessel," which has enabled the detailed study of the migration of macrophages and activated natural killer (NK) cells into tumors in a manner that would have been impossible to see in vivo [11].

\section{Role of Molecular Diagnostics and Sequencing in Immuno-Oncology}

Molecular diagnosis is important for diseases with a genetic component and for understanding the pathomechanisms of various diseases, particularly by use of molecular imaging. Molecular diagnostic technologies are used for the detection of biomarkers, which are also used as diagnostics to guide treatment [12]. Genomic testing is relevant to immuno-oncology.

\section{Genomic Testing}

Genomic testing of tumors may be used for assessing genes in a more global sense, such as the assessment of the quantity of mutations in a tumor. Assessments of mutations in specific genes that inactivate the immune system in tumors such as the STK11 gene are more useful. A study has identified STK11/LKB1 alterations as the most prevalent genomic driver of primary resistance to $\mathrm{PD}-1$ axis inhibitors in KRAS-mutant lung adenocarcinoma. Genomic profiling of TMB may enhance the predictive utility of PD-L1 expression and facilitate personalized combination immunotherapy approaches for genomically defined subsets of lung adenocarcinoma [13].

TMB may be used as a biomarker for ICI therapy as patients who have cancers with a high TMB expression are more likely to benefit from immunotherapy because higher numbers of tumor mutations can increase the presence of neoantigens, which can enhance an antitumor immune response. Whole-exome sequencing of non-small-cell lung cancers (NSCLCs) treated with an anti-PD-1 pembrolizumab showed that higher nonsynonymous TMB was associated with improved objective responses, durable clinical benefits, and progression-free survival. Efficacy also correlated with the molecular smoking signature, higher neoantigen burden, and DNA repair pathway mutations [14]. Analysis of several tumor types has shown a correlation between TMB and response rates to ICI therapy [15]. TMB is predictive of response to therapy in combination therapy as a phase III trial showed that progression-free survival was significantly longer with first-line nivolumab plus ipilimumab compared with chemotherapy among patients with advanced NSCLC and a high TMB [16]. Another study has shown that TMB and PD-L1 expression are independent variables, and a composite of TMB plus PD-L1 further evaluates benefits of ICIs [17]. This study also found that targeted NGS accurately estimates TMB and its elevation indicates likelihood of benefit of ICIs. TMB did not correlate with the expression of PD-L1; both variables had similar predictive capacity. Thus, incorporation of the expression of both TMB and PD-L1 into multivariable predictive models should result in greater predictive power. A study on patients with anti-PD-1/PD-L1-treated advanced NSCLC concluded that combining PD-L1 and TMB as well as somatic copy number alteration (both obtained by targeted sequencing) as biomarkers improved the prediction accuracy of ICI and response compared with using individual factors [18].

A problem with the use of TMB as a biomarker is the large number of assays with lack of standardization. Biomarkers for TMB and PD-L1 expression have identified distinct subgroups in a phase II trial of nivolumab plus low-dose ipilimumab in patients with metastatic NSCLC [19].

\section{Gene Expression Signatures}

ICIs produce durable responses in some melanoma patients, but many patients derive no clinical benefit. A single-cell RNA sequencing on melanoma tumors and computational analyses to detect malignant cell states that promote immune evasion led to identification of a resistance program expressed by malignant cells that is associated with $\mathrm{T}$ cell exclusion and immune evasion [20]. The program is expressed prior to immunotherapy, characterizes cold niches in situ, and predicts clinical responses to anti-PD-1 therapy in an independent cohort of melanoma patients. This study also suggests new therapeutic strategies to overcome immunotherapy resistance.

An 18-gene mRNA expression signature of interferongamma responsive genes in tumor samples of patients correlates with clinical benefit of pembrolizumab; validation studies are being performed in ongoing trials of pembrolizumab [21]. In another study, durable clinical responses to atezolizumab (a PD-L1 inhibitor) were observed in patients with tumors expressing high PD-L1 
Fig. 1. Interrelationship of technologies for

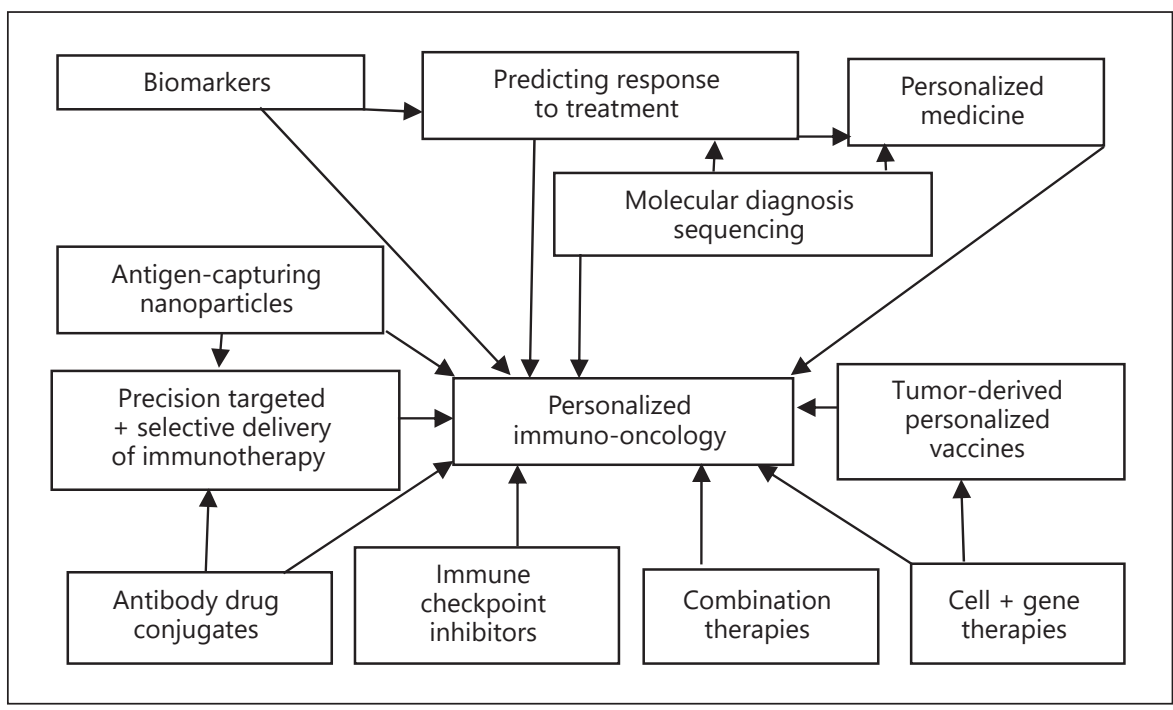
personalized immuno-oncology.

levels on either tumor cells alone or immune cells alone [22]. Thus, PD-L1 expression on tumor cells or immune cells can independently attenuate anticancer immunity, emphasizing the functional importance of immune cells in regulating the antitumor $\mathrm{T}$ cell response.

\section{Personalizing Immuno-Oncology}

Cancers use multiple strategies to counteract anticancer immunity, for example, by selecting cancer cells with immunosuppressive activity through the process of cancer immuno-editing. Anticancer immune responses can also be inhibited in several ways that vary from patient to patient. Therefore, cancer immunotherapy needs to be personalized with the aim of identification of rate-limiting steps in an individual patient and combination of strategies to overcome these hurdles [23].

\section{Integration of Technologies for Personalized Immuno-Oncology}

Figure 1 shows integration of technologies for personalized immuno-oncology.

\section{Factors That Drive the Development of Personalized Immunotherapy for Cancer}

Various factors that drive the development of personalized immuno-oncology are as follows:
- Search for better treatments due to limited efficacy and toxicity of chemotherapy.

- Advances in application of omics technologies in cancer.

- Increasing application of molecular diagnosis and sequencing in cancer.

- Use of cancer biomarkers for diagnosis, as drug targets and to monitor the effects of treatment.

- Combination of diagnostics with therapeutics.

- Advances in understanding the pathophysiology of cancer.

- Availability of biological therapies for cancer.

- Technologies for targeted delivery of anticancer therapeutics.

- Advances in cancer vaccine technologies.

\section{Computational Methods for Personalized Immuno- Oncology}

Multidimensional data derived from patients with cancer from complex laboratory and imaging tests that characterize the molecular underpinnings of an individual patient's cancer require computational methods to analyze these data for facilitating cancer treatment decisions. To address these issues, some important recommendations were made at a workshop [24]:

- Creation and implementation of a computational precision oncology system require multidisciplinary team that should include pathologists, oncologists, pharmacologists, mathematicians, statisticians, and health information technology specialists. 
- There is a need for well-designed prospective clinical trials as well as calibration of machine learning algorithms.

- Criteria are required for evaluating computational tools and deciding when a software product is ready for introduction into clinical practice.

- Evaluation of accuracy of quantitative imaging may also involve an assessment of statistical linearity, which is a measure of how change in the reference reflects proportional change in the measurement and indicates how well measurements reflect clinical realities.

\section{Prediction of Response to Anti-PD-1/PD-L1 Therapy in Cancer}

To determine the key variables for prediction of the response to anti-PD-1/PD-L1 therapy across different cancer types, several neoantigen-, checkpoint-, and immune response-related variables have been systematically evaluated using data from whole-exome and RNA sequencing of patients from the publicly available Cancer Genome Atlas and the objective response rate from a collection of clinical trials [25]. Estimated CD8+ T cell abundance was the most predictive of the response to antiPD-1/PD-L1 therapy across cancer types, followed by the TMB and high PD-1 gene expression. A combination of these 3 variables is highly correlated with $>80 \%$ of the variation in response across different tumor types.

\section{ICls for Cancer}

Cells in the human body have proteins to regulate the immune system response to foreign invaders, for example, cancer cells. Cancer cells can co-opt these "checkpoint" proteins and thwart the immune system's ability to recognize and attack cancer cells. ICIs enable T cells to recognize cancer cells as foreign and prevent deactivation of an immune system response.

\section{Checkpoint Inhibitors Blocking PD-1 or PD-L1}

The PD-1, a regulatory receptor found on T cells, and $\mathrm{PD}-\mathrm{L} 1$, a receptor ligand that binds to $\mathrm{PD}-1$, are both found abundantly on cancer cells. Six drugs, all monoclonal antibodies (MAbs), targeting either PD-1 or PD-L1 have been approved by FDA to treat 14 types of cancer [26]:
Nivolumab can be used to treat melanoma, NSCLC, advanced renal cell carcinoma, Hodgkin lymphoma, squamous cell cancer of the head and neck, and urothelial cancer.

- Pembrolizumab for the treatment of NSCLC.

- Cemiplimab for the treatment of NSCLC.

- Durvalumab for the treatment of advanced or metastatic urothelial carcinoma.

- Atezolizumab for the treatment of urothelial carcinoma.

- Avelumab for the treatment of metastatic Merkel cell carcinoma.

An alternative mechanism of PD-L1 action is described, which involves its secretion in tumor-derived exosomes; removal of exosomal PD-L1 inhibits tumor growth, even in models that are resistant to anti-PD-L1 antibodies [27]. Anti-PD-L1 antibodies act synergistically, not redundantly, with exosomal PD-L1 blockade to suppress tumor growth and could overcome resistance to current antibody approaches.

Clustered regularly interspaced short palindromic repeats (CRISPR)-based screening in experimental studies has identified another mechanism by which cancer cells can regulate the production of $\mathrm{PD}-\mathrm{L} 1$ and suppression of antitumor immunity as the effects of heme inhibition can be replicated by overexpressing a single gene, eIF5B, which is upregulated in human NSCLC with important therapeutic implications [28]. The authors suggest that hampering heme production turns on a pathway called the integrated stress response that cells broadly use to deal with diverse stress conditions such as hypoxia, toxins, or nutrient starvation. Under these conditions, NSCLC cells used a specialized mechanism, relying on a protein known as eIF5B, to increase $\mathrm{PD}-\mathrm{L} 1$ production.

A systematic review and meta-analysis of 19 randomized clinical trials comparing PD-1 and PD-L1 suggest that anti-PD-1 showed favorable survival outcomes and a safety profile comparable to that of anti-PD-L1 in various cancer types, which may provide a useful guide for clinicians [29].

\section{Blockade of the Cytotoxic T-Lymphocyte-Associated Protein 4 by Antibodies}

Antibodies to cytotoxic T-lymphocyte-associated protein 4 (CTLA-4) expressed on T cells are considered to act by checkpoint blockade. In an animal experimental study, a clinically effective anti-CTLA-4 MAb was shown to cause tumor rejection by mechanisms that are indepen- 
dent of checkpoint blockade but dependent on the host $\mathrm{Fc}$ receptor $(\mathrm{FcR})$. These data call for a reappraisal of the CTLA-4 checkpoint blockade hypothesis and provide new insights for the next generation of safe and effective anti-CTLA-4 MAb [30]. Ipilimumab is a MAb that binds to and blocks the activity of CTLA-4, an immune checkpoint protein that is expressed on the surface of T cells. It is approved for the treatment of advanced melanoma.

A significant drawback for choosing ICIs is the low response rate and the problem of resistance to monotherapy. An incentive for the continuing development of novel checkpoint blockade immunotherapies is that only a fraction of patients responds to PD-L1 or CTLA- 4 blockade. Combination of ICIs with other approaches may help address these problems.

\section{Checkpoint Inhibitors Other Than Anti-PD-L1}

ICIs other than anti-PD-L1 have been tested in model systems and have shown a similar inhibitory effect on $\mathrm{T}$ cells to that downstream of PD-1. Lymphocyte activation gene-3 (LAG-3), B- and T-lymphocyte attenuator, programmed death-1 homolog, $\mathrm{T}$ cell immunoglobulin and immunoreceptor tyrosine-based inhibitory motif domain/carcinoembryonic antigen cell adhesion molecule 1 , and the poliovirus receptor-like receptors are some of the receptors that $\mathrm{T}$ cells use to downregulate their response to antigen and are potential therapeutic targets [31]. Like PD-L1, the ligands to these receptors are found on the surface of tumor cells and/or APCs, suggesting that inhibition of TIL via these pathways occurs within tumors. Most of these ICIs are at a preclinical stage but LAG-3 is in clinical trials. IMP321, a soluble LAG-3 protein, has completed phase I and phase II trials as a single agent, as a vaccine adjuvant to improve the anticancer immune response, and in combination with chemotherapy. A phase IIa trial of IMP321 in metastatic melanoma showed that it was well tolerated and induced sustained immune responses [32]. LAG525, an anti-LAG-3 MAb, is in a phase II trial both as a single agent and in combination with PDR-001, a PD-1 inhibitor, for advanced solid tumors (NCT02460224).

\section{Personalizing ICI Therapy}

Immune checkpoint blockade has been used successfully to treat many types of cancer and the response usually correlates with TMB. Additional biomarkers as pre- dictors of response include clonal mutations, alterations in specific genes or signaling pathways, or tumor aneuploidy, but their utility is uncertain in some situations. Results of a recent study reveal genomic correlates of response beyond mutational burden, including somatic events in individual driver genes, and specific HLA-restricted neoantigens, which were often interrelated, indicating the complexity of identifying genetic events that generate an immunoresponsive tumor environment [33]. This study shows that integrated analysis of data from large clinical studies is useful for predicting response to immune checkpoint blockade.

There are ongoing efforts to identify and validate biomarkers to better identify patients who will likely benefit from ICI therapy. For example, expression of PD-L1 in tumor cells (in at least $50 \%$ of cells) correlates with improved efficacy of pembrolizumab [34]. Pembrolizumab improves overall survival compared with chemotherapy as first-line treatment for patients with advanced lung cancer whose PD-L1 expression level is $>1 \%$ [35].

Anti-PD-1 therapy is predicted to enhance T cell cytotoxic activity, as demonstrated by an increased number of TILs and elevated Zap70-pY493 expression [36]. This assay is being used for pharmacodynamic evaluations in ongoing immunotherapy clinical trials.

\section{MAbs for Personalized Management of Cancer}

The remarkable specificity of MAbs enables pairing them with powerful toxins to create specific poisoning agents that seek out cancer cells and kill them. The ability to make MAbs against antigens expressed by human cells has facilitated the identification not only of lymphocytes with different subset functions but also of different antigens on tumor cells. MAbs have several potential targets for cancer. MAbs are also used for diagnosis to identify the source of a tumor and provide the possibility for combining it with personalized treatment.

\section{Antibody-Drug Conjugates for Personalized Therapy of Cancer}

Attaching a cytotoxic "payload" to an antibody to form an antibody-drug conjugate (ADC) provides a mechanism for selective delivery of the cytotoxic agent to cancer cells via the specific binding of the antibody to cell surface receptors on a solid tumor. The development of 2 classes of highly potent microtubule-disrupting agents, maytan- 
sinoids and auristatins, as payloads for ADCs resulted in approval of brentuximab vedotin for treating Hodgkin lymphoma and anaplastic large cell lymphoma, and approval of ado-trastuzumab emtansine for treating HER2positive breast cancer.

Active research on the ADC approach, with $>60 \mathrm{ADCs}$ currently under clinical evaluation, mostly targets solid tumors, for example, TNBC and glioblastoma. The level of target expression is a key parameter in predicting the likelihood of patient benefit for all these ADCs, as well as for the approved compound, ado-trastuzumab emtansine. The development of a patient selection strategy linked to target expression on the tumor is thus critically important for identifying the population appropriate for receiving treatment with an ADC [37].

\section{Cell-Based Immune Therapies}

In humoral immunity, antibodies, which are soluble molecules produced by B lymphocytes, eliminate extracellular pathogens. In cellular immunity, T lymphocytes are triggered to eliminate foreign intracellular pathogens and cancer cells. It is now recognized that many cancer patients have tumor-specific $\mathrm{T}$ cell and antibody responses. Cellular immunotherapy consists of giving the patient cells that stimulate antitumor activity in the patient (tumor and dendritic cell [DC] vaccines) or that have intrinsic antitumor activity (autologous and allogeneic lymphocytes). The aim is to harness potent immunological weapons to destroy cancer cells.

Cytotoxic T lymphocytes can lyse tumor cells. Receptors on the surface of $\mathrm{T}$ cells recognize antigens presented as peptide fragments on the surface of class I major histocompatibility complex (MHC) molecules. As the recognition of an antigen by a naive $\mathrm{T}$ cell bearing an appropriate $\mathrm{T}$ cell receptor is inadequate for activation of the $\mathrm{T}$ cell, the antigen must be encountered in conjunction with a costimulatory signal. In the absence of this, $\mathrm{T}$ cells become tolerant to the antigen.

During the past decade, several tyrosine kinase inhibitors, for example, Bcr-Abl inhibitor imatinib (Glivec ${ }^{\mathrm{TM}}$ ), started to enter the clinic as anticancer agents targeted at the underlying molecular changes in malignant phenotypes. Increasing evidence has emerged to indicate that these drugs exert profound immunomodulatory effects on T cells and APCs, such as DCs, which play major roles in immune tumor surveillance and the outcome of hematopoietic stem cell transplantation. Targeted tyrosine ki- nase inhibitor therapy may thus control cancer cell growth both directly and indirectly by changing the immunological microenvironment.

\section{Treatments for Cancer by ex vivo Mobilization of Immune Cells}

Current cancer therapies successfully treat $50 \%$ of patients, often with serious side effects. The body's T cells and NK cells need to be mobilized against the cancer. This mobilization is done by DCs, specialized cells of the immune system. These cells patrol the body in search of foreign cells such as cancer cells. Once cancer cells are detected, the DCs absorb part of the foreign cells' structure and pass this information on to T cells and NK cells, which are then mobilized to kill the cells.

Although NK cells have long been known to mediate antigen-independent tumor cytotoxicity, the therapeutic potential of NK cell-based immunotherapy has yet to be realized. Manipulating the balance between inhibitory and activating NK receptor signals, sensitization of tumor target cells to NK cell-mediated apoptosis, and recent discoveries in NK-cell receptor biology have fueled translational research that has led to clinical trials investigating a number of novel methods to potentiate NK cytotoxicity against human malignancies.

\section{Granulocytes as Anticancer Agents}

In laboratory experiments, granulocytes from some individuals are more effective in fighting cancer than others. Chemotherapy-induced granulocytopenia can be treated with an approved granulocyte colony-stimulating factor. Granulocyte transfusion has previously been used to try to prevent infections in cancer patients whose immune systems have been weakened by chemotherapy. Granulocytes have been shown to possess cancer-fighting properties, and cells from mice immune to cancer can be used to cure other mice with cancer. The work raises the prospect of using granulocytes from donors to significantly boost the ability of cancer patient to fight their disease, and possibly get cured. The strength of a person's immune system to combat cancer varies according to how stressed the person is and the time of year. It may be possible to transfer granulocytes, which have demonstrated strong cancer-fighting powers, into cancer sufferers. The FDA has given permission to inject superstrength granulocytes into patients in an open-label phase 
I/II clinical trial. The technique, if approved, can be quickly introduced into the clinic because the technology used to extract granulocytes is the same as that already used by hospitals to obtain other blood components such as plasma or platelets. The use of live cells carries a theoretical risk of graft-versus-host disease, which can be fatal. Measures for minimizing this risk are being considered.

\section{Neutrophil Granulocytes in MAb-Based Immunotherapy of Cancer}

MAb can be designed to specifically target tumor-associated antigens and initiate several effector mechanisms, which potentially lead to elimination of cancer. MAbs interact with FcR that are expressed on immune cells. Neutrophils are the most abundant circulating FcRexpressing white blood cells and are equipped with potent cytotoxic ability that is enhanced in the presence of antitumor MAbs. They also play a role in regulating adaptive immunity, which may lead to the initiation of anticancer immune responses. Considerable scientific data support the possibility of exploiting neutrophils for MAb-based immunotherapy of cancer, and an understanding of this may enable future development of new anticancer therapies.

\section{Adoptive Cell Transfer}

ACT, also called adoptive immunotherapy, is the isolation of antigen-specific T lymphocytes, their ex vivo expansion and activation, and subsequent administration in large numbers to the autologous host. This promising approach for inducing antitumor immune responses is a form of personalized cancer therapy. The molecular identification of tumor antigens and the ability to monitor the persistence and transport of transferred cells have provided new insights into the mechanisms of tumor immunotherapy. Several studies have shown the effectiveness of adoptive cell therapy for the treatment of patients with selected metastatic cancers.

\section{Chimeric Antigen Receptor-T Cells}

Chimeric antigen receptors (CAR)- $\mathrm{T}$ cells combine the antigen binding site of a MAb with the signal-activating machinery of a $\mathrm{T}$ cell, freeing antigen recognition from $\mathrm{MHC}$ restriction and thus breaking one of the barriers to more widespread application of cell therapy. CAR-T technology uses retroviral or lentiviral vectors or vectors derived from the Sleeping Beauty transposon/ transposase system to engineer CARs, which graft an arbitrary specificity onto an immune effector cell such as a $\mathrm{T}$ cell. These modified $\mathrm{T}$ cells are then transferred to the patient.

T cells expressing CARs are highly targeted like MAbs but also offer the potential benefits of active trafficking to tumor sites, in vivo expansion, and long-term persistence. Furthermore, gene transfer in T cells enables the introduction of countermeasures to tumor immune evasion and of safety mechanisms [38]. CAR-T cells combine the exquisite antigen specificity of antibodies with the polyfunctionality and potency of cellular immunity, and are a prime example of the potential for synthetic biology to treat cancer. CAR-T cells overcome several of the biologic obstacles that have historically hampered cancer immunotherapy, but the process of manufacturing CARs is more complex than making a pill. Different kinds of cancers require different targets and finding good targets can be difficult. The tools offered by synthetic biology have helped, in some cases, to facilitate the development of CARs. Swapping and testing of various intracellular signaling domains and CAR domains like Lego bricks have become relatively routine experiments being performed in laboratories in academic centers in the USA. Ultimately, extensive clinical trials are required to determine the best engineered domains and study their safety and efficacy.

In 2017, the FDA-approved CTL019 (Kymriah [Tisagenlecleucel]; Novartis), a customized CAR-T gene therapy to treat children and young adults with B-cell acute lymphoblastic leukemia (ALL). It is based on engineering the patient's own T cells, so that when re-introduced into the patient, they attack B cells, which become malignant in ALL. However, the treatment also kills healthy B cells, leaving patients susceptible to infections, so the patients also receive immunoglobulins every few months to bolster their immune systems. In a clinical trial, $83 \%$ of the patients treated were cancer-free after 3 months. This is the first cell/gene therapy to be approved in the USA.

Before CD19-directed CAR T cell therapy, the response rate of available and experimental agents in multiply relapsed and refractory pediatric B-ALL and aggressive B-non-Hodgkin lymphoma was $20-40 \%$, and response duration was short [39]. Tisagenlecleucel definitively improved this outcome in pediatric B-ALL, with minimal residual disease-negative complete re- 
sponses. The ZUMA-1 study has a median of 27.1 months of follow-up, and 39 (39\%) of 101 patients treated remain in response at 2 years (ZUMA-1 studies) [40]. Without such treatment, the response would be an estimated $26 \%$, with only $7 \%$ of patients achieving a complete response. These therapies carry risks related to the activation and expansion of immune effector cells on reinfusion, and $20-30 \%$ of patients require admission to the intensive care unit.

CAR-T cells can be endowed with additional potent functions for improving their antitumor activity, which need to be tightly regulated because they could be harmful without controls. Immune pathways have a wide array of tightly regulated genes that can be repurposed to express potent functions in a highly controlled manner. This concept has been explored by repurposing TCR, CD25, and PD-1, 3 major players of the T cell activation pathway, by inserting the CAR into the TCRa gene (TRACCAR) and IL-12P70 into either IL2R $\alpha$ or PDCD1 genes, resulting in transient, antigen concentration-dependent secretion of IL-12P70, increased TRACCAR T cell cytotoxicity, and extended survival of tumor-bearing mice [41].

\section{Cytokines}

Cytokines (IL-2, IL-12, and granulocyte-macrophage colony-stimulating factor [GM-CSF]) are small glycoproteins that bind to the surface receptors on immune cells and regulate their function and survival. Cytokine treatment is the oldest active immuno-oncology therapy and was based on the infusion of cytokines to activate the patient's own immune system. Interferon- $\alpha$, an approved cytokine treatment, activates NK cells and causes tumor cell death. IL-2, originally approved as monotherapy for metastatic renal cell carcinoma and metastatic melano$\mathrm{ma}$, is now being investigated in combination with other cytokines, cell-based immunotherapies, chemotherapeutic agents, ICIs, and vaccines.

\section{Personalized Cancer Vaccines}

Cancer vaccines are of different types: nucleic acidbased, MAb-based, and cell-based vaccines. Most of the personalized cancer vaccines are cell-based, and these were the earliest forms of personalized medicine as patient's own cells are used [42]. Various types of cells used include tumor cells and DCs.

\section{Autologous Tumor Cell Vaccines}

Whole tumor cells, rendered safe by irradiation and mixed with an immunological adjuvant, were one of the earliest forms of cellular therapy. This approach avoids the need for tumor antigens to be identified before treatment and allows all the relevant antigens to be included in the vaccine. Initial clinical studies showed the safety of this approach, with side effects mainly limited to local reactions at the site of the vaccine injection. Immunogenicity of tumor cell vaccines can be improved by transducing the tumor cell with genes that encode key components of the immune response (cytokines such as GM-CSF and costimulatory molecules). Because individual cancers within the same pathological category are antigenically distinct requires that each patient's immune system be trained to recognize that patient's specific cancer. This is the basis of manufacture of cancer immunotherapeutics from each patient's own tumor tissue. Another approach is to identify as many candidates as possible for tumorassociated T cell epitopes in individual patients. Expression profiling of tumor and normal tissue can be performed to identify genes exclusively expressed or overexpressed in the tumor sample.

Mass spectrometry enables characterization of several different MHC ligands from the same tumor sample. Combining these 2 analytic tools, it is possible to propose several candidates for peptide-based immunotherapy. This integrated functional genomics approach can be used for the design of antitumor vaccines tailored to suit the needs of each patient.

Whole tumor vaccines have gone through clinical trials. Melacine consists of lysed cells from 2 human melanoma cell lines combined with a proprietary adjuvant. It is approved for metastatic melanoma in Canada. None of the tumor cell vaccines are in the market in the USA. Recent evidence suggests that the best source of antigens for vaccines is autologous tumor because $T$ cells that target tumor neoantigens arise from cancer mutations, which are the mediators of several effective cancer immunotherapies in humans such as cytokine IL-2, ACT, and ICIs [43]. Immunogenomic studies have enabled identification of nonsynonymous mutations, determination of messenger sequences that can be transcribed as well as translated, and prediction of the neoantigenicity and MHC-binding potential of specific molecules [44]. Therefore, the best way to present such autologous tumor antigens may be on autologous DCs rather than directly injecting antigens.
10

Med Princ Pract 2021;30:1-16 DOI: $10.1159 / 000511107$
Jain 


\section{DC-Based Cancer Vaccines}

DC-based cancer vaccines are a major focus in cancer immunotherapy as the primary functions of DCs are the initiation and regulation of immune responses. Although several subsets of DC have been identified in humans, the 2 most common subsets are myeloid DCs recognized by their expression of either CD1c or CD141 and plasmacytoid DC identified by their expression of CD123 and CD303. Some tumors may stop responding to DC-based vaccines due to the development of immune tolerance, which can be overcome by personalized DC-based cancer vaccines as they contain nearly all the antigens in a tumor. Antigen-specific approaches may generate an antigen-specific response even when the tumor antigens are not known.

Cancer vaccines target tumor mutations because they are not found in healthy tissue, but every person's cancer has its own unique set of mutations, which makes a generalized vaccine for all types of a cancer impossible. The most promising approach would be patient-tailored, and may use a combined approach: antigen-specific as well as tumor-derived. However, some tumors may stop responding to DC-based vaccines due to the development of immune tolerance, which can be overcome by personalized DC-based cancer vaccines as they contain nearly all the antigens in a tumor. In clinical trials on malignant melanoma, patient-specific vaccines consisting of autologous DCs loaded with antigens from irradiated cells from short-term autologous tumor cell lines were associated with encouraging survival [45]. Combination with chemotherapy may also be helpful by elimination of cancer cells and inhibition of tumor-induced suppressive factors.

\section{Cytokines as Vaccine Adjuvants}

Cytokines are also used as natural adjuvants of vaccines of various formulations to help in activating and maintaining an antitumor immune response. IL-12, secreted by immune cells in response to an invading pathogen, can also alert $T$ cells to recognize, attack, and remember tumor cells for future. IL-12 or its gene is most effective when injected directly into tumors, not infused into the bloodstream as much higher concentration of IL-12 when applied directly to tumors prompts cells to express specific genes at higher levels and/or transmit signals along different anti-proliferation or suicide-inducing pathways. Thus, the identification of specific genes and pathways might enable the amplification of anticancer effects with IL-12 or other compounds.

Personalized Immuno-Oncology

\section{Limitations of Cancer Vaccination}

Several clinical trials of cancer vaccines have been conducted with a high failure rate. Reasons for failure include the following:

- The immune system is already damaged by chemotherapy in some patients and may not respond to vaccines.

- Vaccines based on a single antigen are less effective than those that raise an immune response against a broad range of tumor antigens to minimize the chance of the tumor becoming resistant to the therapy.

- Immune responses to vaccines may take a few months to evolve, and tumors that grow rapidly may outpace it.

- Some cancer patients with advanced and bulky tumors are not good subjects for immunotherapy.

\section{Role of Neoantigens in Personalizing Cancer Vaccines}

Mutated proteins expressed only in cancer cells and recognizable by the immune system are known as neoantigens. Tumor neoantigens are "foreign" proteins not found in normal human organs/tissues. They are specifically recognized by neoantigen-specific TCRs in the context of MHC molecules. Neoantigens play a critical role in tumor-specific T-cell-mediated antitumor immune response and successful cancer immunotherapies. Neoantigen-based therapeutic personalized vaccines and adoptive $\mathrm{T}$ cell transfer have shown promising preliminary results [46]. Examples of mutation-induced neoantigen-specific activation of $\mathrm{T}$ cells include lung cancer, head and neck squamous cell carcinoma, colorectal cancer, breast cancer, and lymphomas [47]. The genetic landscape of human cancer and genomic information obtained by NGS has enabled identification of such candidate neoantigens in individual cancers. Future cancer vaccines will require personalization by identification of both patient-specific immunosuppressive mechanisms and target neoantigens [23].

\section{Immunogene Therapy}

One of the major goals of cancer immunotherapy is the induction of tumor-specific $\mathrm{T}$ lymphocyte responses that will be effective in the rejection of established tumors. The prospects for this therapy rely on the identification of tu- 
mor antigens. Gene therapy technology enables new approaches, in which vectors are designed to deliver gene combinations for the expression of proteins directed against different targets and mechanisms. Strategies of immunogene therapy may include several overlapping techniques:

- Ex vivo therapies involving the use of tumor cells transduced with genes for immunostimulatory molecules using viral or nonviral vectors.

- In vivo immunogene therapy: direct injection of cytokine gene vectors into tumors, naked DNA injection, and RNAi as well as antisense inhibition of immunosuppressive function.

- In situ genetic engineering of tumors for long-lasting and systemic immunotherapy.

- Cancer vaccines.

- MAb gene transfer.

- Gene-editing tools such as to remove alloreactivity and decrease immunogenicity of third-party $\mathrm{T}$ cells.

A novel approach is based on synthetic, biodegradable nanoparticles as vectors that can genetically reprogram cancer cells and their microenvironment in situ, so that the cancer cells can act as tumor-associated APCs by inducing immunostimulation [48]. Use of these nanoparticles in combination with checkpoint blockade led to a systemic cytotoxic immune response with significant reduction of tumor growth in mouse cancer models. The advantages of this approach are as follows:

- Local nanoparticle delivery avoids adverse side effects of systemic administration of immunotherapeutics.

- Gene delivery is achieved without use of viruses that can have safety concerns.

- Immunotherapy response is achieved without requiring previous knowledge of tumor-expressed antigens.

- Systemic anticancer response reduces the chances of recurrence or metastases.

\section{Delivery of Cancer Immunotherapy}

Immune-related adverse events due to the low specificity of tumor cell targeting strongly limit immunotherapy efficacy. Engineered delivery systems and controlled release innovations are in development to improve $\mathrm{MAb}$ accumulation and retention within target cells and tissues for enhancing immunotherapeutic efficacy and reducing off-target effects [49]. Efficacy of immunotherapeutic MAbs, usually given intravenously, is influenced by the route of administration. In preclinical models, intratumoral, peritumoral, and subcutaneous injection have been shown to improve efficacy both by enhancing delivery of MAb locally to the tumor and to reduce toxicity by reducing systemic accumulation. Nanobiotechnology enables delivery of different immunotherapeutic agents specifically to the tumor site, thus increasing efficacy and reducing toxicity.

\section{Nanoparticles for Delivery of Immunotherapy Combined with Photodynamic Therapy}

Available in different materials, nanoparticles can be formulated with tailored shape, charge, size, and surface chemical modifications to make them suitable for individual applications [50]. Positron emission tomography (PET)-guided photodynamic therapy (PDT) with biodegradable mesoporous silica nanoparticles (bMSNs) can be used for personalized cancer immunotherapy as they accumulate in tumor after intravenous injection [51].

PDT is an effective, noninvasive therapeutic modality against local tumors that are accessible to the source of light. However, the application of PDT for the treatment of disseminated metastatic cancer remains challenging. On the other hand, cancer immunotherapy offers a promising approach for generating systemic antitumor immune responses against disseminated cancer. A multifunctional nanomaterial system has been described for the combination of PDT and personalized cancer immunotherapy and their potency demonstrated against local as well as disseminated tumors [50]. Specifically, uniform and bMSNs with an average size of $\sim 80 \mathrm{~nm}$ and large pore size of 5-10 nm were synthesized for theranostic PETguided PDT and neoantigen-based cancer vaccination. Multiple neoantigen peptides, $\mathrm{CpG}$ oligodeoxynucleotide adjuvant, and photosensitizer chlorin e6 were coloaded into a bMSN nanoplatform, and PET imaging revealed effective accumulation of bMSN in tumors (up to $9.0 \% \mathrm{ID} / \mathrm{g}$ ) after intravenous administration. Subsequent PDT with laser irradiation recruited DCs to PDT-treated tumor sites and elicited neoantigen-specific, tumor-infiltrating cytotoxic T cell lymphocytes. Using multiple murine models of bilateral tumors, the authors demonstrated strong antitumor efficacy of PDT-immunotherapy against locally treated tumors as well as distant, untreated tumors. These findings suggest that the bMSN is a promising platform for combining imaging and PDT-enhanced personalized immunotherapy for the treatment of advanced cancer. 


\section{Synthetic Bacteria for Local Tumor Delivery of Checkpoint Blockade Nanobodies}

Synthetic biology has been used for targeted delivery of nanobodies to increase anticancer efficacy of check point inhibitor therapy. Bacteria, prepared on the basis of an optimized lysing mechanism coupled with immunotherapeutic expression for the intratumoral delivery of nanobodies targeting PD-L1 and CTLA-4, showed higher therapeutic efficacy than analogous antibodies in producing regression of tumors in syngeneic mouse models [52]. An enhancement of a systemic immune response was observed, with a relative increase in activated $\mathrm{T}$ cells and corresponding increases in systemic T cell memory populations in mice treated with this approach. Therapeutic efficacy was further enhanced by combination with a probiotically produced GM-CSF.

\section{Transdermal Cold Atmospheric Plasma-Mediated ICI Therapy}

Despite the promise of ICI therapy against cancer, challenges associated with low objective response rates and severe systemic side effects limit its clinical applications. Cold atmospheric plasma (CAP)-mediated ICI therapy, integrated with hollow-structured microneedle transdermal patch, facilitates the transportation of CAP through the skin, for delivery of ICB and lead to death of tumor cells [53]. The release of tumor-associated antigens then promotes the maturation of DCs in the tumordraining lymph nodes, subsequently initiating T-cell-mediated immune response. Anti-PD-L1 antibodies released from the transdermal patch further augment antitumor immunity. These findings indicate that ICI therapy with transdermal CAP can inhibit the growth of both primary and distant tumors, prolonging the survival of tumorbearing mice.

\section{Combination of Immunotherapies for Cancer}

The most common type of combination is a PD-1/PDL1 drug with another immunotherapy drug, but PD-1/ PD-L1 drugs are also being evaluated in combination with targeted therapies, for example, chemotherapy and radiation therapy. Other examples of combination therapies are as follows:

1. CTLA-4 and PD-1 have a synergistic effect; simultaneous blocking of both improves the clinical anticancer response as their inhibitory signals block cancer at multiple steps and in multiple cell types. Administration of anti-PD-1 antibodies in combination with antibodies targeting CTLA-4 in malignant melanoma increased clinical responses rate up to $50 \%$ but at cost of higher treatment-related toxicities [54].

2. Combination of BRAF inhibitors as the standard therapy was replaced by combination of BRAF inhibitor and MEK inhibitor and now single anti-programmed cell death protein DNA methyltransferase inhibitors can be combined with ICIs, such as those that block CTLA-4 and PD-1 [55].

3. In mouse models of cancer, combination of anti-angiogenesis and PD-1 ICI is synergistic and decrease the expression of multiple immune checkpoints more than PD-1 alone [56].

A study using mass cytometry and single-cell RNA sequencing of tumors that respond relatively well to immune checkpoint therapy and those that do not, a unique population of CD73hi macrophages was identified in glioblastoma that persists after anti-PD-1 treatment [57]. The authors suggest a combination therapy strategy to target CD73 with anti-CD73 antibodies, which have yielded promising results in clinical studies, plus dual blockade of PD-1 and CTLA-4 to improve the response to immune checkpoint therapy in glioblastoma.

Immunomodulatory activities of epigenetic drugs that lead to improved immune recognition of cancer cells have the potential for improving the anticancer activity of novel immunotherapies through combinatorial epigenetic immunotherapy approaches [58]. Findings of an animal experimental study indicate that combined histone deacetylase (HDAC)6 inhibition and immune checkpoint blockade represents a potential treatment strategy for ARID1A-mutated cancers, for example, ovarian carcinoma [59]. These findings provide a rationale for combining epigenetic modulators and existing immunotherapeutic interventions. Clinical trials are in progress to test different combinations of immunotherapy and epigenetic therapy in various cancers to find a perfect match of treatment, that is, personalized cancer therapy for patients.

Combination of ICIs or anti-PD-1/PD-L1 inhibitor and anti-CTLA-4 inhibitor might replace the singleagent therapy [60]. Clinical trials of combinatorial approaches of neoantigen-based therapies with other immunotherapies such as ICIs as well as conventional anticancer treatments are ongoing. For example, a phase I trial combines neoantigen-based personalized vaccine with ICI therapy in patients with newly diagnosed glio- 
blastoma. Combination strategies for evaluation in clinical trials will be prioritized [61]. A cross-sectional study analyzed results from 98 clinical trials testing PD-1 pathway inhibitors alone or in combination with other agents in patients with metastatic cancer to identify optimal combinations of PD-1 ICIs with other cancer therapies [62]. Most combination trials involving PD-1 or PD-L1 ICIs resulted in objective response rates greater than that expected from PD-1 monotherapy associated with those inhibitors.

\section{Concluding Remarks and the Future of Personalized Immuno-Oncology}

Personalized/precision approach is generally well accepted for management of cancer. Immuno-oncology, one of the most promising approaches to management of cancer, fits in well with the principles of personalized medicine. Molecular diagnostics is an important basis of personalized medicine, and genomic technologies along with NGS play an important role in immuno-oncology. Similarly, there is an emphasis on biomarker research both in validating current biomarkers and in the identification and development of new biomarkers - to improve selection of patients who might benefit from ICI therapy.
Considerable progress has been made in immuno-oncology in the past decade. It has enabled treatment of aggressive cancers that could not be managed with conventional anticancer therapies and has provided long-term survival benefits. Over 4,000 methods of treatment, mostly drugs, are in development. Most of these are still at a preclinical stage. Cell therapies have shown the greatest growth.

Clinical trials have shown that neoantigen-based vaccines do elicit some $\mathrm{T}$ cell responses with few severe adverse side effects and when combined with PD-1 checkpoint inhibitors, trial participants receiving both had more durable responses than with either therapy alone [63]. In personalized immune therapy, there is a choice of several types of tumor-associated antigens as well as neoantigens by direct injection that can be used as target antigens to enable $\mathrm{T}$ cells to destroy tumor cells [64]. According to these authors, other methods of administration include peptide-loaded DCs in vaccination approaches or infusion of ex vivo expanded tumor-specific $\mathrm{T}$ cells, but such cell therapies have potential problems such as immune suppressive tumor microenvironment, lack of persistence of ex vivo expanded antigen-specific $\mathrm{T}$ cells, and potential off-target toxicity.

\section{Conflict of Interest Statement}

The author has no conflict of interest to declare.

\section{References}

1 Jain KK. Textbook of personalized medicine. 3rd ed. Cham: Springer; 2021.

2 Akiki M, Haddad FG, Kourie HR, Khaddage A, Smayra VT, Smayra VT. PD-L1: an unavoidable biomarker in advanced triplenegative breast cancer. Biomark Med. 2019; 13(18):1539-41.

3 Music M, Prassas I, Diamandis EP. Optimizing cancer immunotherapy: Is it time for personalized predictive biomarkers? Crit Rev Clin Lab Sci. 2018;55(7):466-79.

4 Navas T, Fino K, Fung KL, Cutuli F, Kinders RJ, Sharma A, et al. A multiplex immunofluorescence assay to assess immune checkpoint inhibitor-targeted CD8 activation and tumor colocalization in FFPE tissues. J Clin Oncol. 2019;37(15_Suppl 1):2629.

5 Castaneda CA, Castillo M, Aliaga K, Bernabe LA, Casavilca S, Sanchez J, et al. Level of tumor-infiltrating lymphocytes and density of infiltrating immune cells in different malignancies. Biomark Med. 2019;13(17): 1481-91.

6 Kroeze LI, de Voer RM, Kamping EJ, von Rhein D, Jansen EAM, Hermsen MJW, et al.
Evaluation of a hybrid capture-based pancancer panel for analysis of treatment stratifying oncogenic aberrations and processes. J Mol Diagn. 2020;22(6):757-69.

7 Jenkins RW, Aref AR, Lizotte PH, Ivanova E, Stinson S, Zhou CW, et al. Ex vivo profiling of PD-1 blockade using organotypic tumor spheroids. Cancer Discov. 2018;8(2): 196-215.

8 Narasimhan V, Das A, Pham T, Wilson K, Kong J, Ramsay R, et al. Organoids: the new kid in cancer research. ANZ J Surg. 2019; 89(10):1189-90.

9 Pauli C, Hopkins BD, Prandi D, Shaw R, Fedrizzi T, Sboner A, et al. Personalized in vitro and in vivo cancer models to guide precision medicine. Cancer Discov. 2017;7(5): 462-77.

10 Boussommier-Calleja A, Li R, Chen MB, Wong SC, Kamm RD. Microfluidics: a new tool for modeling cancer-immune interactions. Trends Cancer. 2016;2(1):6-19.

11 Polini A, Del Mercato LL, Barra A, Zhang YS, Calabi F, Gigli G. Towards the development of human immune-system-on-a-chip platforms. Drug Discov Today. 2019;24(2): 517-25.

12 Jain KK. A handbook of biomarkers. 2nd ed. New York: Humana/Springer; 2017.

13 Skoulidis F, Goldberg ME, Greenawalt DM, Hellmann MD, Awad MM, Gainor JF, et al. STK11/LKB1 mutations and PD-1 inhibitor resistance in KRAS-mutant lung adenocarcinoma. Cancer Discov. 2018;8(7):82235.

14 Rizvi NA, Hellmann MD, Snyder A, Kvistborg P, Makarov V, Havel JJ, et al. Cancer immunology. Mutational landscape determines sensitivity to PD-1 blockade in nonsmall cell lung cancer. Science. 2015; 348(6230):124-8.

15 Yarchoan M, Hopkins A, Jaffee EM. Tumor mutational burden and response rate to PD-1 inhibition. N Engl J Med. 2017; 377(25):2500-1.

16 Hellmann MD, Ciuleanu TE, Pluzanski A, Lee JS, Otterson GA, Audigier-Valette C, et al. Nivolumab plus ipilimumab in lung cancer with a high tumor mutational burden. $\mathrm{N}$ Engl J Med. 2018;378(22):2093-104. 
17 Rizvi H, Sanchez-Vega F, La K, Chatila W, Jonsson P, Halpenny D, et al. Molecular determinants of response to anti-programmed cell death (PD)-1 and anti-programmed death-ligand 1 (PD-L1) blockade in patients with non-small-cell lung cancer profiled with targeted next-generation sequencing. J Clin Oncol. 2018;36(7):633-41.

18 Kim HS, Cha H, Kim J, Park WY, Choi YL, Sun JM, et al. Genomic scoring to determine clinical benefit of immunotherapy by targeted sequencing. Eur J Cancer. 2019;120:6574.

19 Ready N, Hellmann MD, Awad MM, Otterson GA, Gutierrez M, Gainor JF, et al. Firstline nivolumab plus ipilimumab in advanced non-small-cell lung cancer (CheckMate 568): outcomes by programmed death ligand 1 and tumor mutational burden as biomarkers. J Clin Oncol. 2019;37(12):9921000.

20 Jerby-Arnon L, Shah P, Cuoco MS, Rodman C, Su MJ, Melms JC, et al. A cancer cell program promotes $\mathrm{T}$ cell exclusion and resistance to checkpoint blockade. Cell. 2018; 175(4):984-e24.

21 Ayers M, Lunceford J, Nebozhyn M, Murphy E, Loboda A, Kaufman DR, et al. IFN$\gamma$-related mRNA profile predicts clinical response to PD-1 blockade. J Clin Invest. 2017; 127(8):2930-40.

22 Music M, Prassas I, Diamandis EP. Optimizing cancer immunotherapy: Is it time for personalized predictive biomarkers? Crit Rev Clin Lab Sci. 2018;55(7):466-79.

23 Kakimi K, Karasaki T, Matsushita H, Sugie T. Advances in personalized cancer immunotherapy. Breast Cancer. 2017;24(1):1624.

24 National Academies of Sciences, Engineering \& Medicine. Improving cancer diagnosis and care: clinical application of computational methods in precision oncology: proceedings of a workshop. Washington, DC: The National Academies Press; 2019.

25 Lee JS, Ruppin E. Multiomics prediction of response rates to therapies to inhibit programmed cell death 1 and programmed cell death 1 ligand 1. JAMA Oncol. 2019;5(11): 1614-8.

26 Tang J, Yu JX, Hubbard-Lucey MV, Neftelinov ST, Murphy E, Loboda A, et al. The clinical trial landscape for PD1/PDL1 immune checkpoint inhibitors. Nat Rev Drug Discov. 2018;17(12):854.

27 Poggio M, Hu T, Pai C-C, Chu B, Belair CD, Chang A, et al. Suppression of exosomal PDL1 induces systemic anti-tumor immunity and memory. Cell. 2019;177(2):414-27.

28 Suresh S, Chen B, Zhu J, Golden RJ, Lu C, Evers BM, et al. eIF5B drives integrated stress response-dependent translation of PD-L1 in lung cancer. Nat Cancer. 2020; 1(5):533-45.

29 Duan J, Cui L, Zhao X, Bai H, Cai S, Wang $G$, et al. Use of immunotherapy with programmed cell death 1 vs programmed cell death ligand 1 inhibitors in patients with cancer: a systematic review and meta-analysis. JAMA Oncol. 2019;6(3):375-84.

30 Du X, Tang F, Liu M, Su J, Zhang Y, Wu W, et al. A reappraisal of CTLA-4 checkpoint blockade in cancer immunotherapy. Cell Res. 2018;28(4):416-32.

31 Torphy RJ, Schulick RD, Zhu Y. Newly emerging immune checkpoints: Promises for future cancer therapy. Int $\mathrm{J}$ Mol Sci. 2017;18(12):E2642.

32 Legat A, Maby-El Hajjami H, Baumgaertner P, Cagnon L, Maillard SA, Geldhof C, et al. Vaccination with LAG-3Ig (IMP321) and peptides induces specific CD4 and CD8 Tcell responses in metastatic melanoma patients - report of a phase I/IIa clinical trial. Clin Cancer Res. 2016;22(6):1330-40.

33 Miao D, Margolis CA, Vokes NI, Liu D, Taylor-Weiner A, Wankowicz SM, et al. Genomic correlates of response to immune checkpoint blockade in microsatellite-stable solid tumors. Nat Genet. 2018;50(9):127181.

34 Garon EB, Rizvi NA, Hui R, Leighl N, Balmanoukian AS, Eder JP, et al. Pembrolizum$\mathrm{ab}$ for the treatment of non-small-cell lung cancer. N Engl J Med. 2015;372(21):201828.

35 Mok TSK, Wu YL, Kudaba I, Kowalski DM, Cho BC, Turna HZ, et al. Pembrolizumab versus chemotherapy for previously untreated, PD-L1-expressing, locally advanced or metastatic non-small-cell lung cancer (KEYNOTE-042): a randomised, open-label, controlled, phase 3 trial. Lancet. 2019; 393(10183):1819-30.

36 Navas T, Fino K, Fung KL, Cutuli F, Kinders RJ, Sharma A, et al. A multiplex immunofluorescence assay to assess immune checkpoint inhibitor-targeted CD8 activation and tumor colocalization in FFPE tissues. J Clin Oncol. 2019;37(15_Suppl 1):2629.

37 Lambert JM, Morris CQ. Antibody-drug conjugates (ADCs) for personalized treatment of solid tumors: a review. Adv Ther. 2017;34(5):1015-35.

38 Ramos CA, Dotti G. Chimeric antigen receptor (CAR)-engineered lymphocytes for cancer therapy. Expert Opin Biol Ther. 2011;11(7):855-73.

39 Crump M, Neelapu SS, Farooq U, Van Den Neste E, Kuruvilla J, Westin J, et al. Outcomes in refractory diffuse large B-cell lymphoma: results from the international SCHOLAR-1 study. Blood. 2017;130(16): $1800-8$

40 Locke FL, Ghobadi A, Jacobson CA, Miklos DB, Lekakis LJ, Oluwole OO, et al. Longterm safety and activity of axicabtagene ciloleucel in refractory large B-cell lymphoma (ZUMA-1): a single-arm, multicentre, phase 1-2 trial. Lancet Oncol. 2019;20(1):31-42.

41 Sachdeva M, Busser BW, Temburni S, Jahangiri B, Gautron AS, Maréchal A, et al. Repurposing endogenous immune pathways to tailor and control chimeric antigen recep- tor T cell functionality. Nat Commun. 2019; 10(1):5100

42 Jain KK. Personalized cancer vaccines. Expert Opin Biol Ther. 2010;10(12):1637-47.

43 Tran E, Robbins PF, Rosenberg SA. Final common pathway of human cancer immunotherapy: targeting random somatic mutations. Nat Immunol. 2017;18(3):255-62.

44 Hundal J, Miller CA, Griffith M, Griffith OL, Walker J, Kiwala S, et al. Cancer immunogenomics: computational neoantigen identification and vaccine design. Cold Spring Harb Symp Quant Biol. 2016;81:105-11.

45 Dillman RO, Cornforth AN, McClay EF, Depriest C. Patient-specific dendritic cell vaccines with autologous tumor antigens in 72 patients with metastatic melanoma. Melanoma Manag. 2019;6(2):MMT20.

46 Jiang T, Shi T, Zhang H, Hu J, Song Y, Wei $\mathrm{J}$, et al. Tumor neoantigens: from basic research to clinical applications. J Hematol Oncol. 2019;12(1):93.

47 Yang W, Lee KW, Srivastava RM, Kuo F, Krishna C, Chowell D, et al. Immunogenic neoantigens derived from gene fusions stimulate T cell responses. Nat Med. 2019; 25(5):767-75.

48 Tzeng SY, Patel KK, Wilson DR, Meyer RA, Rhodes KR, Green JJ. In situ genetic engineering of tumors for long-lasting and systemic immunotherapy. Proc Natl Acad Sci U S A. 2020;117(8):4043-52.

49 Francis DM, Thomas SN. Progress and opportunities for enhancing the delivery and efficacy of checkpoint inhibitors for cancer immunotherapy. Adv Drug Deliv Rev. 2017;114:33-42.

$50 \mathrm{Xu}$ C, Nam J, Hong H, Xu Y, Moon JJ. Positron emission tomography-guided photodynamic therapy with biodegradable mesoporous silica nanoparticles for personalized cancer immunotherapy. ACS Nano. 2019; 13(10):12148-61.

51 Iscaro A, Howard NF, Muthana M. Nanoparticles: properties and applications in cancer immunotherapy. Curr Pharm Des. 2019;25(17):1962-79.

52 Gurbatri CR, Lia I, Vincent R, Coker C, Castro S, Treuting PM, et al. Engineered probiotics for local tumor delivery of checkpoint blockade nanobodies. Sci Transl Med. 2020; 12(530):eaax0876

53 Chen G, Chen Z, Wen D, Wang Z, Li H, Zeng Y, et al. Transdermal cold atmospheric plasma-mediated immune checkpoint blockade therapy. Proc Natl Acad Sci U S A. 2020;117(7):3687-92.

54 Paschen A, Schadendorf D. The era of checkpoint inhibition: Lessons learned from melanoma. Recent Results Cancer Res. 2020;214:169-87.

55 Dear AE. Epigenetic modulators and the new immunotherapies. N Engl J Med. 2016; 374(7):684-6.

56 Yi M, Jiao D, Qin S, Chu Q, Wu K, Li A. Synergistic effect of immune checkpoint blockade and anti-angiogenesis in cancer treatment. Mol Cancer. 2019;18(1):60. 
57 Goswami S, Walle T, Cornish AE, Basu S, Anandhan S, Fernandez I, et al. Immune profiling of human tumors identifies CD73 as a combinatorial target in glioblastoma. Nat Med. 2020;26(1):39-46.

58 Maio M, Covre A, Fratta E, Di Giacomo AM, Taverna P, Natali PG, et al. Molecular pathways: at the crossroads of cancer epigenetics and immunotherapy. Clin Cancer Res. 2015; 21(18):4040-7.

59 Fukumoto T, Fatkhutdinov N, Zundell JA, Tcyganov EN, Nacarelli T, Karakashev S, et al. HDAC6 inhibition synergizes with antiPD-L1 therapy in ARID1A-inactivated ovarian cancer. Cancer Res. 2019;79(21): 5482-9.
60 Lee DH. Practical issues of biomarker-assisted targeted therapy in precision medicine and immuno-oncology era. ESMO Open. 2018;3(Suppl 1):e000370.

61 National Academies of Sciences, Engineering, and Medicine. Advancing progress in the development of combination cancer therapies with immune checkpoint inhibitors: proceedings of a workshop. Washington DC: The National Academies Press; 2019.
62 Schmidt EV, Chisamore MJ, Chaney MF, Maradeo ME, Anderson J, Baltus GA, et al. Assessment of clinical activity of PD-1 checkpoint inhibitor combination therapies reported in clinical trials. JAMA Netw Open. 2020;3(2):e1920833.

63 Ott PA, Hu Z, Keskin DB, Shukla SA, Sun J, Bozym DJ, et al. An immunogenic personal neoantigen vaccine for patients with melanoma. Nature. 2017;547(7662):217-21.

64 Bastien JP, Minguy A, Dave V, Roy DC. Cellular therapy approaches harnessing the power of the immune system for personalized cancer treatment. Semin Immunol. 2019;42:101306 